\title{
Nonequilibrium Interface Kinetics During Rapid Solidification
}

\section{Citation}

Aziz, Michael J. 1987. Nonequilibrium interface kinetics during rapid solidification. Materials Research Society Symposium Proceedings 74: 25-38.

\section{Published Version}

http://www.mrs.org/s_mrs/sec.asp?CID=1727\&DID=38980

\section{Permanent link}

http://nrs.harvard.edu/urn-3:HUL.InstRepos:2839213

\section{Terms of Use}

This article was downloaded from Harvard University's DASH repository, and is made available under the terms and conditions applicable to Other Posted Material, as set forth at http:// nrs.harvard.edu/urn-3:HUL.InstRepos:dash.current.terms-of-use\#LAA

\section{Share Your Story}

The Harvard community has made this article openly available.

Please share how this access benefits you. Submit a story.

\section{Accessibility}




\title{
NONEQULIBRIUM INTERFACE KINETICS DURING RAPID SOLIDIFICATION
}

\author{
MICHAEL J. AZIZ \\ Division of Applied Sciences, Harvard University, Cambridge MA 02138
}

\begin{abstract}
The deviations from local equilibrium at a rapidly moving solid-liquid interface are well documented. The fraction of solute atoms in the liquid at the interface that joins the crystal during rapid solidification approaches unity and the interface temperature drops. Experimental and theoretical work on impurity incorporation and interfacial undercooling is reviewed. Past and future experiments to test the theories are discussed.
\end{abstract}

\section{INTRODUCTION}

Rapid solidification experiments have reached a crystal growth regime where deviations from local equilibrium are obvious and interface motion is no longer strictly heat-flow limited. These experiments allow us the opportunity to study the interface reaction kinetics in high * mobility systems for the first time. In the first part of this paper, I review evidence for the departure from local equilibrium. I then describe a simple model, applicable to both metals and semiconductors, for the kinetics of the fundamental atomic processes occurring at the interface during rapid solidification of binary alloys. The result is a pair of "interface response functions" which predict (a) how much solute should be incorporated into the solid, and (b) how fast the interface should move; in terms of the local conditions at the interface, namely undercooling and composition. I then describe some recent experiments regarding question (a) that until recently have been possible only in semiconductors; the results support one model and rule out others. Finally, the predictions of a model for question (b) are presented for an ideal solution and for a simple eutectic system.

Consider what happens when a liquid alloy is cooled very slowly, as shown in Fig. 1(a). If the cooling rate is so slow that compositional equilibrium can be maintained throughout the bulk of the liquid and the solid phases, then the phase diagram tells us what we need to know: a solid of uniform composition given by the equilibrium solidus will coexist with a liquid of uniform composition given by the equilibrium liquidus. As the temperature decreases, the fraction of solid material increases according to the lever rule.

Under normal processing conditions, this scenario never occurs. Rather, composition gradients in the solid and often also in the liquid are created and are governed by the laws of long-range mass transport. It is still possible, however, that the atoms in each phase immediately adjacent to the interface still have enough time to establish a local equilibrium across the interface itself. With this popular assumption of local equilibrium, the phase diagram is used as an interface condition diagram as in Fig. 1(b). That is, although the composition is nonuniform throughout the bulk of the two phases, given a composition of the liquid at the interface, the solid grows at the equilibrium temperature, with the equilibrium solidus composition.

Now during rapid solidification, the composition of the solid at the interface can usually be inferred afterward from the composition of the bulk of the solid, but the interface temperature and the liquid composition at the interface are of ten unknown. The local equilibrium assumption survives only if the interface temperature was on the solidus curve or its metastable extension during quenching, as shown in Fig. 1(c). The assumption of local equilibrium was proved invalid 17 years ago by Baker and $\mathrm{Cahn}$ [1], who splat-quenched a series of $\mathrm{Zn}$-Cd alloys to obtain solid solutions containing Cd concentrations greater than the retrograde composition in Fig. I(d). To this day, we are still unsure about what to use in place of the equilibrium phase diagram to predict the conditions at a rapidly-moving interface. In this paper, a proposed model for a kinetic interface condition diagram, which reduces to the equilibrium phase diagram at slow interface velocities, will be described.

Pulsed laser melting of doped semiconductors $[2,3]$ permitted experiments under very wellcontrolled conditions; for example with one-dimensional experimental geometries, allowing simple but accurate calculations, and even measurements, of important quantities such as impurity depth profiles and interface positions and velocities. Heat-flow calculations and 

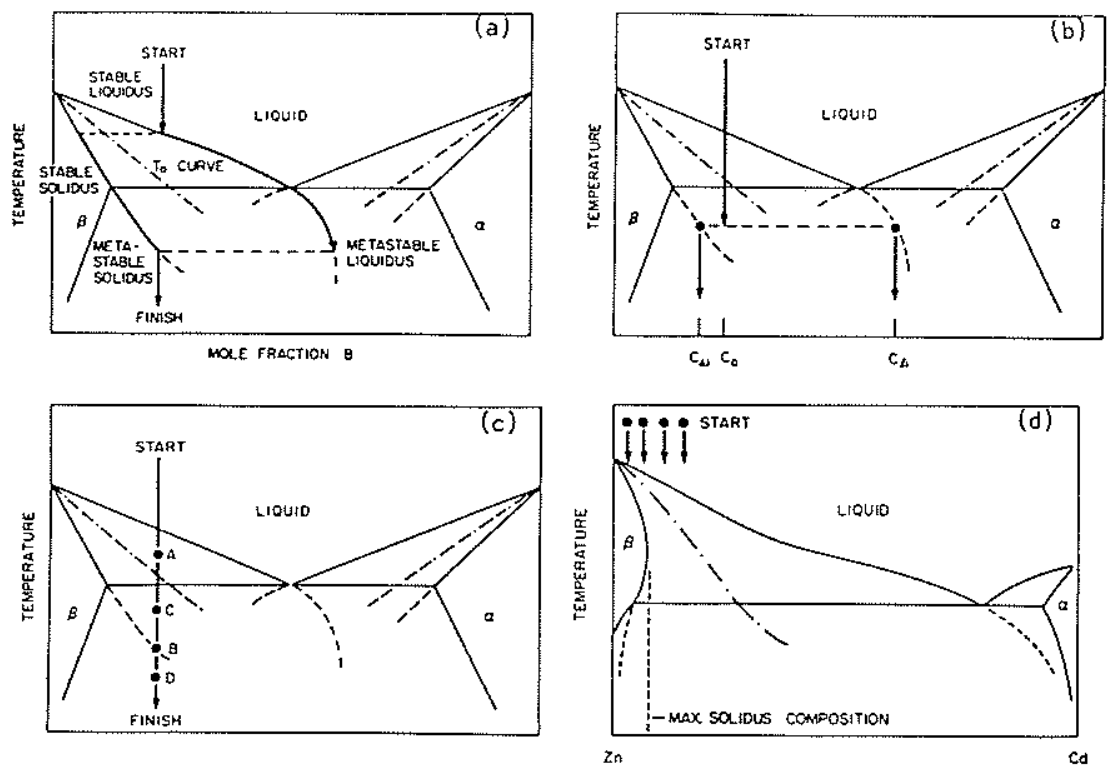

Fig. I. Interface conditions during solidification. (a) Metastable global equilibrium. (b) Local equilibrium. (c) Potential interface temperatures during rapid solidification. Point A: impossible, Point B: local equilibrium, Points $C$ and D: possible, no local equilibrium. (d) Baker-Cahn experiment proving local equilibrium to be invalid.

post-irradiation analyses of dopant distributions showed that maximum equilibrium solubilities could be exceeded by several orders of magnitude, and that the partition coefficient $k$, defined by the ratio of the impurity concentration in the growing solid to that in the liquid at the interface, departs from its equilibrium value and approaches unity. In systems with retrograde solidi it could be shown that the impurity had actually undergone an increase in chemical potential upon solidification, termed "solute trapping" $[1,4]$.

A number of physically plausible models have been proposed [5-13] to explain how the host atoms persuade the impurity atoms to increase their chemical potential and join the crystal. They were in qualitative agreement with the early data, which show the partition coefficient monotonically increasing from its equilibrium value $\mathrm{k}_{\mathrm{e}}$ toward unity as the interface speed, $\mathrm{v}$, increases.

The basis for the model described here is based on an idea due to Tumbull viz., that crystallization and interdiffusion at the interface involve processes that are fundamentally different. Conventional modeling of interface kinetics [10] uses the same atomic motions to describe crystallization as those to describe interdiffusion at the solid-liquid interface. In such a case, the growth speed can never exceed the maximum diffusive speed, which is typically on the order of $1-10 \mathrm{~m} / \mathrm{s}$, given by $v_{D}=D_{i} / \lambda$, where $D_{i}$ is the coefficient for interdiffusion across (not along) the interface and $\lambda$ is the interatomic distance. However, the collision-limited growth model developed by Tumbull and coworkers [14-22], which has received recent support from the molecular dynamics work of Broughton et al. [23] and the picosecond laser melting experiments of MacDonald et al. [24] on pure metals, indicate that for metallic and other simple molecular systems such a scaling is invalid and that $v_{5}$, the speed of sound, should be the only 
limit to the crystal growth rate. Consequently, the growth speed can very much exceed the diffusive speed, and when it does so in alloys, we expect suppressed partitioning and a diffusionless transformation.

Atoms in the interface region tend to interdiffuse in order to establish equilibrium compositions on either side of the interface. As the interface passes, solute-solvent redistribution only occurs during a limited time. Before the interface anives, the driving force for redistribution is zero; afterwards, atomic mobilities are too low to permit significant interdiffusion. When $v \ll v_{D}$, there is plenty of time for the atoms at the interface to equilibrate. As the growth velocity becomes greater, solute and solvent atoms have less time while they are in the interface region to interdiffuse and maintain local equilibrium. When $v>v_{D}$, the atoms have virtually no time to interdiffuse; the interface passes and locks the liquid composition into the bulk of the solid before the atoms have a chance to react.

If growth occurs by the periodic rapid lateral passage of steps of height $\lambda$, then the time between the passage of steps is $t_{c}=\lambda / v$. The time it takes solvent atoms to assume their lattice positions is negligible compared to $t_{\mathrm{c}}$, and the shifts can be treated as virtually instantaneous. On the other hand, if continuous solidification occurs, the impurity atom is being dragged toward its lattice site during a time period of order $t_{c}$. An analysis of solute diffusion back into the liquid [11] during the interval $t_{C}$ between the passing steps in the former case yields, for a dilute solution of $\mathrm{B}$ in $\mathrm{A}$,

$$
k(v, T)=k_{e}(T)+\left[1-k_{e}(T)\right] \exp \left(-v_{D} / v\right)
$$

where the equilibrium partition coefficient $\mathrm{k}_{\mathrm{e}}(\mathrm{T})$ is the ratio of the equilibrium solidus composition to the equilibrium liquidus composition at the temperature $\mathrm{T}$ of the undercooled interface, and the diffusive speed $v_{D}$ is the only fitting parameter in the model. This model should apply when the chemical potential of the solute atom is raised from its liquid value to its solid value during a time interval that is very short compared to $t_{c}$, altemating periodically with diffusive solute escape between such events. In the latter case of continuous growth, in the limit of strict steady-state fluxes on a microscopic scale, the fluxes and concentrations become timeindependent and a similar analysis [12] yields

$$
k(v, T)=\left[\left(v / v_{D}\right)+k_{e}(T)\right] /\left[\left(v / v_{D}\right)+1\right]
$$

which shows the same qualitative features as in stepwise growth, a transition from equilibrium partitioning $\left(k=k_{e}\right)$ to complete solute trapping $(k \rightarrow 1)$ as the interface speed surpasses the diffusive speed. In the continuous growth model, however, the transition is more gradual than in the stepwise growth model. Recently it has been shown [25] that eq. (2) follows also from the stepwise growth model if the interval between the passage of steps is random, rather than fixed.

The dilute solution approximation is invalid when the mole fraction of solute is no longer negligible or when activity coefficients are no longer constants. When the appropriate corrections to the fluxes and driving forces in the continuous growth model are made [26], the result is:

$$
k\left(v, T, X_{L}\right)=\frac{\frac{v}{v_{D}}+\kappa_{o}}{\frac{v}{v_{D}}+1-\left(1-\kappa_{o}\right) X_{L}}
$$


Here $X$ denotes the mole fraction of solute, the partitioning parameter $\kappa_{0}\left(X_{L}, X_{S}, T\right)$ $\equiv \exp \left[-\Delta\left(\mu_{B}^{\prime}-\mu_{A}^{\prime}\right) / R T\right]$ is a measure of the driving force for redistribution, and $\mu^{\prime}(X, T)$ $\equiv \mu(X, T)-R T \ln X$, where $\mu$ is the chemical potential. The symbol $\Delta$ denotes a subtraction of the value of a quantity in the liquid at the interface from the value of that quantity in the solid. The subscripts $S$ and $L$ refer to the solid and the liquid, $A$ and $B$ refer to the solvent and solute.

\section{EXPERIMENTAL RESULTS}

The most significant experimental progress in the fundamentals of interface kinetics during rapid alloy solidification has been made with pulsed laser melting of doped semiconductors. Progress was rapid because defect-free plane-front solidification is routinely attained and because in many cases $k_{e}$ is so small that when $k$ approaches unity tremendous changes are effected in the final impurity distribution.

The pioneering work of White et al. $[2,27]$ and of Baeri et al. $[3,28]$ showed that $k$ can in fact increase several orders of magnitude above $k_{\mathrm{e}}$ at high interface speeds and demonstrated the velocity-dependence of $\mathrm{k}$ by varying the laser and substrate parameters to cover a range of regrowth velocities during pulsed laser melting of ion implanted Si. When compared in detail, the results of the two groups were contradictory in some cases. Certain studies showed an effect termed "saturation", where $\mathrm{dk} / \mathrm{dv} \rightarrow 0$ at some value of $\mathrm{k}<1$. This effect was reported for Bi [28], In [29], and $\mathrm{Sb}$ [30] in $\mathrm{Si}$. In a separate study of $\mathrm{Bi}$ in $\mathrm{Si}$, however, the effect was not observed [27]. This saturation phenomenon is intriguing because it cannot be explained by any

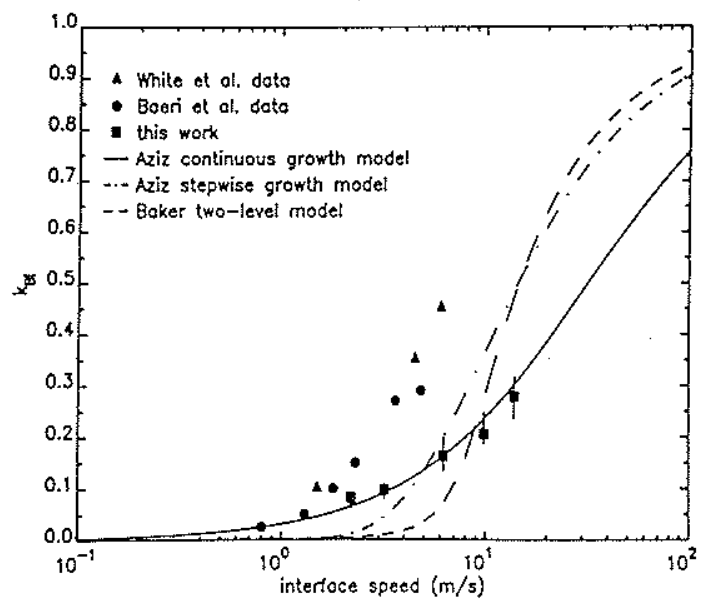

Fig. 2. Dependence of partition coefficient on velocity. Triangles and circles: velocity estimated from heat-flow calculations. Squares: velocity measured by transient conductance. Continuous growth model: $v_{D}=32 \mathrm{~m} / \mathrm{s}$. Stepwise growth model: $v_{D}=10 \mathrm{~m} / \mathrm{s}$. Baker model: $v_{D}=1 \mathrm{~m} / \mathrm{s}$.

simple model; if correct, it indicates that more complicated solute trapping models $[8,13,31]$ are necessary for understanding the phenomenon.

A study was undertaken [32] to examine the saturation phenomenon in detail and to see whether any of the existing models could yield quantitative agreement with the data. To obtain high precision data, we used the transient conductance technique for measuring the interface velocity [33] for Bi-implanted Si, thereby eliminating errors that arose in the previous studies, which could only estimate $\mathrm{v}$ by the use of heat-flow calculations. The time-evolution of the melt depth was measured with roughly 1 ns resolution with this technique, which measures changes in the transverse electrical conductance of a thin film specimen as the phase transformation 
proceeds. The initial and final Bi profiles were determined to high accuracy by Rutherford Backscattering Spectrometry (RBS) carried out in grazing exit angle geometry. Analysis of the partition coefficient was performed by numerical simulation with a finite-element solution of the diffusion equation, using the measured as-implanted profile and the measured melt depth as a function of time for each sample. Impurity diffusion was assumed to occur in the liquid phase, but neglected in the solid phase. During solidification, a fraction $\mathrm{k}$ of the impurity in the liquid at the interface was incorporated into the crystal. The parameters allowed to vary were the bulk liquid-phase diffusivity $\mathrm{D}_{\mathrm{L}}$ and the partition coefficient during solidification $\mathrm{k}$. Comparison with the measured profiles yielded unique values for both $\mathrm{D}_{\mathrm{L}}$ and $\mathrm{k}$.

We show in Fig. 2 the results of analysis of several samples. The predictions of the models discussed above for stepwise growth and for continuous growth are shown, as is a twolevel version of a model due to Baker and Cahn $[8,11,20]$, where the impurity is treated as if diffusing in a continuum down a steep energy gradient at the interface. For this model, $k$ is given by $\mathrm{k}=\left\{\beta+\ln \mathrm{k}_{\mathrm{e}}\right\} /\left\{\beta+\left[\left(1 / \mathrm{k}_{\mathrm{e}}\right)\left(\ln \mathrm{k}_{\mathrm{e}}\right) \exp (-\beta)\right]\right\}$, where $\beta \equiv \mathrm{v} / \mathrm{v}_{\mathrm{D}}$. The diffusive speed $v_{D}$ is the only free parameter in all three models; it has been chosen in each case to yield the best overall fit to the data. Note that in each of these models $v$ only appears as the ratio $v / v_{D}$; thus when plotted as $\mathrm{k}$ versus $\log \mathrm{v}$, as in Fig. 2 , the only effect of varying the free parameter $\mathrm{v}_{\mathrm{D}}$ is a rigid horizontal shift of the curve, with no change in the slope. We see that the stepwise growth model and the two-level Baker model produce curves that rise too steeply to account for our data. The continuous growth model, on the other hand, fits our data quite well. This model seems to account well for the velocity dependence of trapping of other species in $\mathrm{Si}$ as well [34].

Other solute trapping models were not considered. One model due to Jackson and coworkers [10] produces a $\mathrm{k}(\mathrm{v})$ curve similar to that of the continuous growth model discussed here, but has been rejected because it cannot account for solute trapping on both ends of a phase diagram [34]. We are unable to compare our results to the models of Hillert and Sundman [9] and of Gilmer [13] because they involve complex computer calculations that we cannot perform.

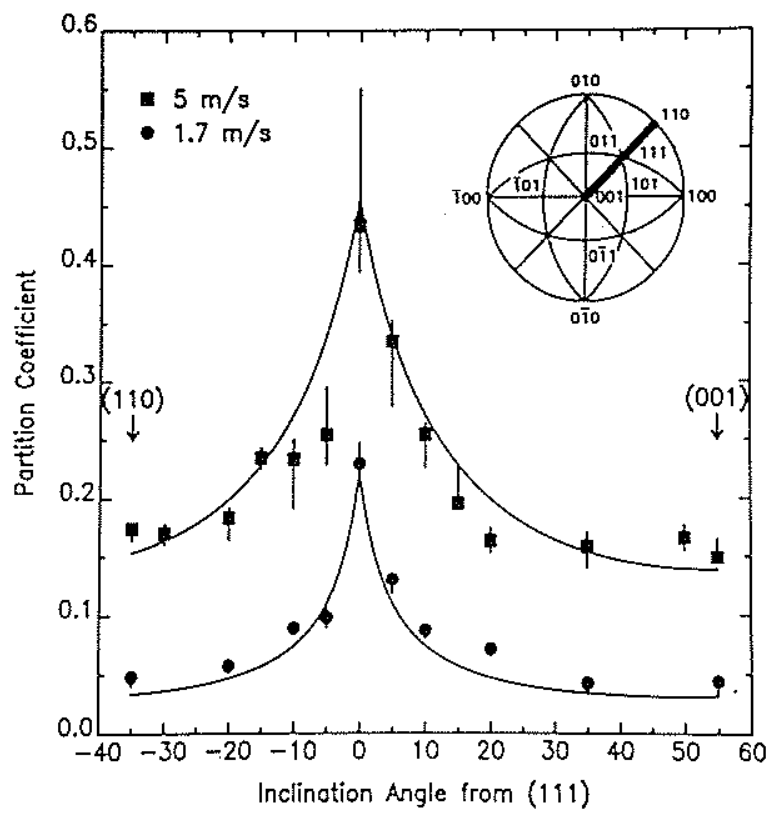

Fig. 3. Orientation dependence of partition coefficient of Bi in Si at two speeds.

Squares: $5 \mathrm{~m} / \mathrm{s}$.

Circles: $1.7 \mathrm{~m} / \mathrm{s}$.

Random Interval Model with $v_{D}{ }^{L}=20 \mathrm{~m} / \mathrm{s}$, $v_{D} T=6 \mathrm{~m} / \mathrm{s}$. Upper curve: $v=5 \mathrm{~m} / \mathrm{s}$, lower curve: $v=1.7$ $\mathrm{m} / \mathrm{s}$. 
Data from the original work of Baeri et al. [28] and White et al. [27] are also shown in Fig. 2. While sufficient to establish that $k$ increases far beyond $k_{e}$ as $v$ increases, we see here that their data were not accurate enough to allow any conclusions regarding appropriate models or the presence of the saturation effect discussed earlier. Note that there is no evidence in our data for such an effect. It appears that the main source of the disagreement is the use in the original heat flow calculations of an assumed thermal conductivity of amorphous Si that is very much greater than measurements eventually indicated [35]. This apparently caused errors in their calculations of the time-evolution of the melt depth, which fed back to produce errors in the apparent $k$.

Orientation dependence. Similar agreement with the continuous growth model was obtained for trapping at the (111) interface. The accuracy was limited by the unavailability of (111) Si-on-sapphire, forcing us to rely on heat-flow calculations of the interface position and velocity. It was surprising to find that the stepwise growth model does not apply even at the (111) interface. A full study of the orientation dependence of $k$ was undertaken [36] to resolve this puzzle.

Si wafers were cut at orientations from (110) through (111) to (001) at $5^{\circ}$ increments and given identical $\mathrm{Bi}$ implants. They were then irradiated with a pulsed excimer laser under two sets of conditions, calculated to yield regrowth speeds of 1.7 and $5 \mathrm{~m} / \mathrm{s}$, respectively. As a check on the calculations and the shot-to-shot variation, the laser pulse energy was monitored by using time-resolved reflectivity to measure the melt duration for each shot. Numerical simulations were performed as above to determine $k$, using the calculated melt depth as a function of time. The results, plotted in Fig. 3, show a sharp peak in $\mathrm{k}$ at the (111) orientation. The Random Interval Model [25], an extension of the simple models presented above, yields the curves in Fig. 3. As shown in Fig. 4, we assume in the new model that at all orientations considered, crystal growth occurs by the lateral passage of (111) steps of monolayer height with lateral speed $v_{L}=v / \sin |\theta|$, where $\theta$ is the inclination angle from (111). The absence of cusps at (110) and (001) in Fig. 3 suggests the absence of ledges having these orientations on surfaces near these orientations. We assume that the solute incorporation and solute escape processes occur at the edge of the moving

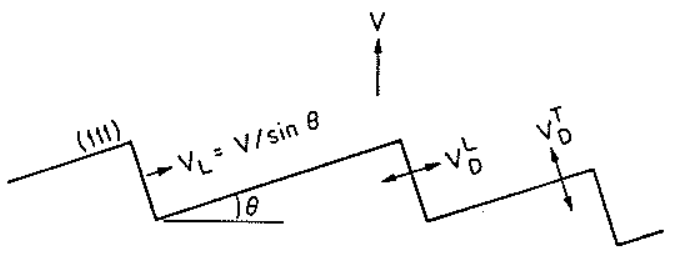

Fig. 4. Solute trapping by lateral passage of ledge, with solute escape flux at step edge and terrace.

step itself. Since from entropic considerations we expect the steps to be heavily kinked and therefore diffuse laterally, the kinetics of continuous lateral ledge motion vs. lateral solute-solvent redistribution lead to the simple continuous growth model (eq. (2)), with $v$ replaced by $v_{L}$ and $\mathrm{v}_{\mathrm{D}}$ replaced by the maximum speed $\mathrm{v}_{\mathrm{D}}{ }^{\mathrm{L}}$ of redistribution across the step edge. Solute atoms then have a second chance to escape back into the liquid through the terrace, characterized by a maximum speed $v_{D}{ }^{T}$ of redistribution across the terrace, before they are covered by the passage of the next growth step. Finally, the passage of subsequent growth steps is assumed to occur randomly in time rather than periodically. (This last assumption is necessary to fit the data for the velocity dependence of $k$.) Since the solute atom in the terrace is triply or quadruply bonded to the crystal, whereas at the ledge it is only doubly bonded to the crystal, we expect $v_{D}{ }^{T}<$ $v_{D}{ }^{L}$. In fact, $v_{D}{ }^{T}$ and $v_{D} L$ are used as the only two fitting parameters in the model. A very good fit to the data for both the velocity and orientation dependence of $k$ is obtained when $v_{D} T=$ $6 \mathrm{~m} / \mathrm{s}$ and $\mathrm{v}_{\mathrm{D}}{ }^{\mathrm{L}}=20 \mathrm{~m} / \mathrm{s}$. 


\section{GROWTH VELOCITY}

Onsager's reciprocity relations [37] imply that if crystal growth affects the amount of solute incorporation, then solute trapping will in tum affect the growth rate. The common assumption of heat-flow-limited growth is in many cases a useful one. It does, however, become unrealistic at high velocities and solute concentrations, where the interface reaction kinetics can come into play. The implications of solute trapping for the crystal growth rate are described below.

For pure systems, the crystal growth process is understood as follows [38]. In equilibrium, the rates at which atoms join and leave the crystal are equal. When there is a finite driving force for crystallization, the former exceeds the latter by Boltzmann factor exp( $-\Delta G / R T)$; the growth velocity is given by their difference. The kinetics are manifested by an intrinsic growth-velocity limit, which is the speed of the forward reaction alone, $v_{0}(T)$. This is the speed with which the interface would propagate at infinite driving force (i.e., when the back reaction disappears). The growth velocity is then written as $v=v_{0}(T)[1-\exp (\Delta G / R T)]$ (note $\Delta G$ is defined to be negative during solidification). This expression becomes the familiar $\mathrm{v}=\mathrm{M} \Delta \mathrm{G}$ at small driving forces, where $M$ is conventionally called the mobility of the interface and $\Delta \mathrm{G}$ is conventionally called the driving force for crystallization. For pure systems, the driving force is the difference in chemical potential $\Delta \mu$. For alloys, the driving force is $\Delta \mathrm{G}_{\mathrm{DF}}=\mathrm{X}_{\mathrm{S}} \Delta \mu_{\mathrm{B}}+(1-$ $\left.\mathrm{X}_{\mathrm{S}}\right) \Delta \mu_{\mathrm{A}}$. An assumption then must be made regarding how the interface velocity is related to $\Delta G_{D F}$. Two different assumptions have been made in this regard. In the first, called "no solute $\mathrm{drag}^{\prime \prime}[11,39]$, an equation of the same form as that for pure systems is used, with $\Delta \mathrm{G}_{\mathrm{DF}}$ replacing $\Delta \mu$ :

$$
\mathrm{v}=\mathrm{v}_{\mathrm{O}}\left[1-\exp (1 / \mathrm{RT})\left(\Delta \mathrm{G}_{\mathrm{DF}}\right)\right]
$$

In the second, termed "with solute drag" $[9,12,40]$, some of $\Delta \mathrm{G}_{\mathrm{DF}}$ is assumed to be dissipated by "solute drag" driving the solute-solvent redistribution reaction and is therefore unavailable to drive the crystallization reaction. In this case, the "solute drag free energy" $\Delta G_{D}$ is subtracted off of $\triangle \mathrm{G}_{\mathrm{DF}}$ :

$$
v=v_{\mathrm{O}}\left[1-\exp (1 / \mathrm{RT})\left(\Delta \mathrm{G}_{\mathrm{DF}}-\Delta \mathrm{G}_{\mathrm{D}}\right)\right]
$$

The free energy dissipated by the crystallization reaction is called $\Delta \mathrm{G}_{\mathrm{C}}$ and must be equal to $\Delta G_{D F}-\Delta G_{D} \cdot \Delta G_{D}$ and thus $\Delta G_{C}$ can be calculated exactly, with no free parameters, for any steady-state solute trapping model [26]. The result is $\Delta \mathrm{G}_{\mathrm{C}}=\mathrm{X}_{\mathrm{L}} \Delta \mu_{\mathrm{B}}+\left(1-\mathrm{X}_{\mathrm{L}}\right) \Delta \mu_{\mathrm{A}}$.

There is a useful construction involving $\Delta \mathrm{G}_{\mathrm{DF}}, \Delta \mathrm{G}_{\mathrm{C}}$, and the free energy curves for the solid and liquid phases, shown in Fig. 5. $\Delta \mathrm{G}_{\mathrm{C}}$ is the vertical distance, at composition $\mathrm{X}_{\mathrm{L}}$, from the liquid curve to the tangent to the solid curve at $X_{S}$, as shown in Fig. 5(a). Given a temperature and a liquid composition, one can use these curves to determine the solid composition when $v$ and $v_{D}$ are small compared to $v_{0}$. For if $v / v_{O}$ is very small then in the model with solute drag, $\Delta \mathrm{G}_{\mathrm{C}}\left(=\Delta \mathrm{G}_{\mathrm{DF}}-\Delta \mathrm{G}_{\mathrm{D}}\right)$ is very small in (4). Thus the vertical arrow in Fig. $5(a)$ is very small. Very little error is involved then in locating the solution to eq. (4) by approximating $X_{S}$ in Fig. $5(\mathrm{a})$ by the point on the solid curve whose tangent intersects the liquid curve at $\mathrm{X}_{\mathrm{L}}$. Likewise, $\Delta \mathrm{G}_{\mathrm{DF}}$ is given by the more familiar construction in Fig. $5(\mathrm{~b})$; it is the vertical distance, at composition $X_{S}$, to the solid curve from the tangent to the liquid curve at $X_{L}$. In the model without solute drag $\Delta \mathrm{G}_{\mathrm{DF}}$ is very small in (3); we locate the solution to (3) by approximating $X_{S}$ in Fig. 5 (b) by the intersection of the solid curve with the tangent from the 

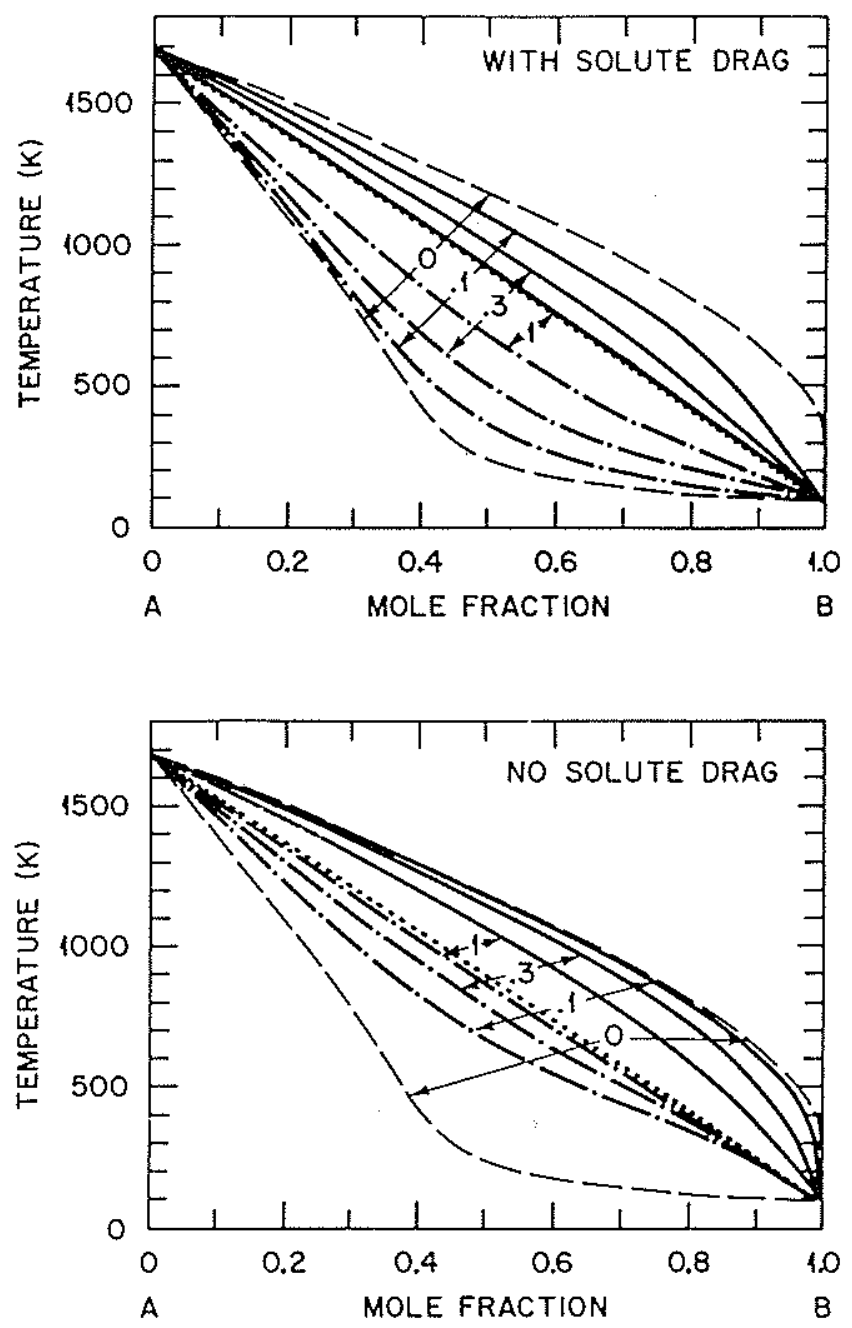

Fig. 6. Kinetic interface condition diagram for ideal solution liquid and solid with melting points as shown, entropy of fusion $=2.3 R$, and $v_{O} / v_{D}=100 .(a)$ : Solutions of eqs. $(2 a)$ and (4) "with solute drag", (b): eqs. (2a) and (3) "without solute drag"; for values of $v / v_{D}$ shown. Dashed lines: solutions for $v=0$ corresponding to equilibrium phase diagram. Dotted line: To curve. Solid line: kinetic liquidus. Dot-dashed line: kinetic solidus. 

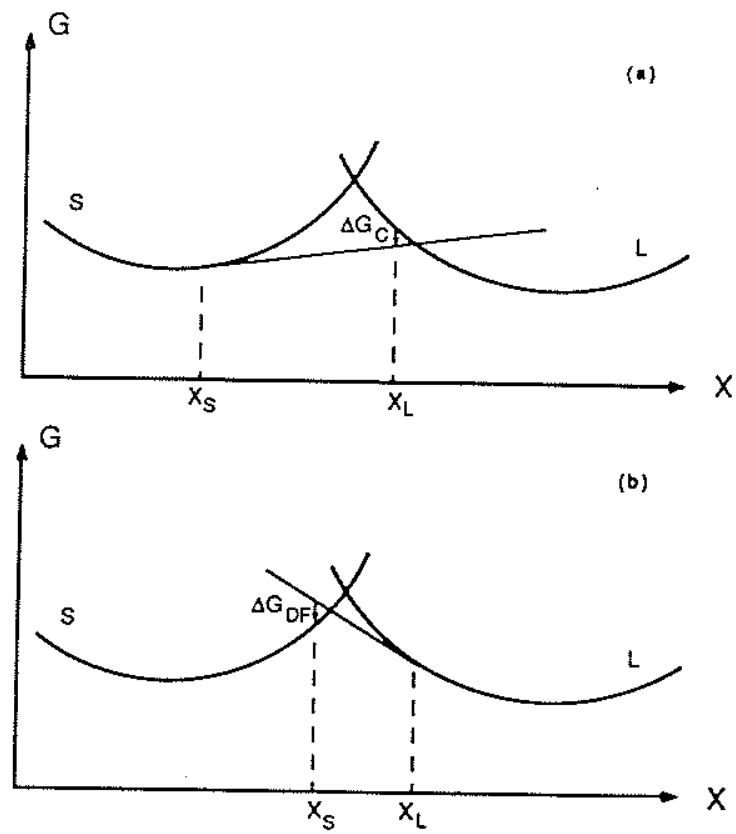

Fig. 5. Construction for obtaining composition of growing solid given temperature and composition of liquid at interface. (a) Model with solute drag, $\Delta G_{C} \rightarrow 0$ as $v_{O} \rightarrow \infty$. (b) Model without solute drag, $\Delta G_{D F} \rightarrow 0$ as $v_{O} \rightarrow \infty$.

liquid curve at $\mathrm{X}_{\mathrm{L}}$. The actual solutions to (3) and (4) lie to the left of the intersections in Figs. 5 (a) and 5 (b), respectively, by amounts that scale with $v / v_{0}$. Note that these constructions are independent of the particular form of the $k(v)$ equation used. We expect the constructions to be quite accurate for materials exhibiting collision-limited growth kinetics, for which $v_{0}$ should be of the order of the speed of sound. The velocity can then be found by inverting a $k(v)$ equation, such as (1), (2), or (2a). The constructions break down by the time $X_{L}$ crosses the $T_{O}$ line, the intersection of the two free energy curves. By this point, the arrows in Figs. 5(a) and 5(b) must be large enough to be drawn to scale on the diagrams. If $v_{D}$ is similar in magnitude to $v_{O}$, then the liquid and solid compositions do not deviate from the local equilibrium compositions until $v$ approaches $v_{O}$, and the range of validity of the construction is quite limited. This is not the case for systems obeying collision-limited growth, calculations for which are presented below. Finally, note from the constructions that in the model with solute drag the liquid composition drops with increasing $\mathrm{v}$ much faster than the solid composition rises, whereas the reverse is true in the model without solute drag.

\section{THEORETICAL RESULTS}

The kinetic model requires as input the chemical potential of each species in each phase at all temperatures and compositions of interest. If we hope to compare the predictions of any kinetic model with experimental results, an accurate thermodynamic model is essential, for if the predictions do not agree with experiment, we must know whether the fault lies in the thermodynamic or in the kinetic model. The results of calculations for ideal solutions in the 

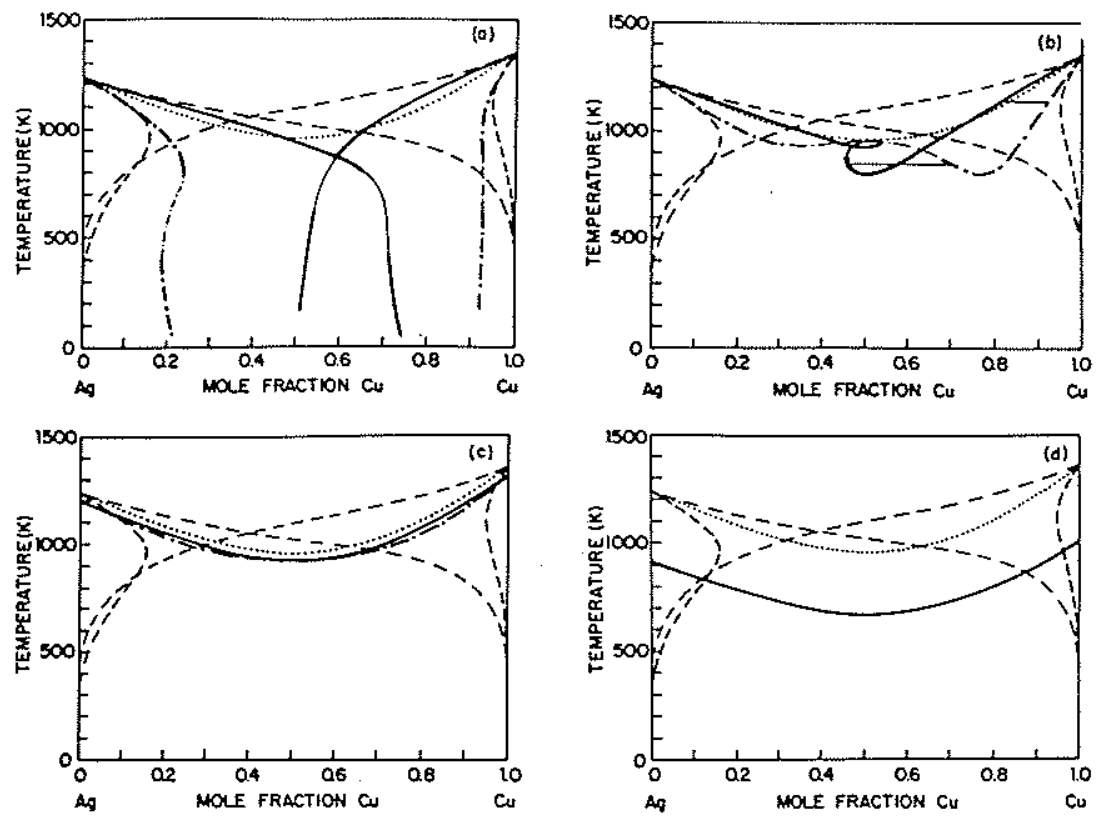

Fig. 7. Kinetic interface condition diagram for Ag-Cu assuming $v_{O} / v_{D}=100$. The effect of solute drag is included. Dashed lines: solutions for $v=0$ corresponding to equilibrium phase diagram. Dotted line: $T_{O}$ curve. Solutions for: $(a) v / v_{D}=0.1$; solid line: kinetic liquidus; dot-dash line: kinetic solidus. Thin regions of kinetic solidus and liquidus denote unstable roots. (b) $v / v_{D}=0.3$; (c) $v / v_{D}=3.0 ;$ (d) $v / v_{D}=30$.

liquid and in the solid are presented first, followed by the results of calculations for the $\mathrm{Ag}-\mathrm{Cu}$ system, a simple eutectic system in which the terminal phases have the same crystal structure. $\mathrm{Ag}-\mathrm{Cu}$ has been modeled thermodynamically throughout the undercooled liquid regime by Murray [41]; we use her model in the calculation. The only free parameters are the speed of interdiffusion at infinite driving force $v_{D}$, and the speed of crystal growth at infinite driving force $v_{0}$. We assume that collision-limited growth describes these systems, and therefore use an estimate of $v_{O} / v_{D}=100$, independent of composition and temperature (in practice, $v_{D}$ may depend more strongly on temperature and composition than does $\mathrm{v}_{\mathrm{O}}$ ).

The four variables $X_{s}, X_{L}, T$, and $v$ are related by the pair of equations (2a) and ( 3 or 4 ). We can thus express any pair of variables in terms of the other pair. We display our results in the interface condition diagrams of Figs. 6-8. They show, for example, the interface temperature and solid composition that result if one imposes a certain velocity and liquid composition at the interface. Alternatively, they show the interface temperature and the liquid composition at the interface that result if one imposes a certain velocity and solid composition on the system, as in steady-state welding [42].

The system in Fig. 6 is an ideal solution in both the liquid and the solid. The melting points are 1700 and $100 \mathrm{~K}$; the molar entropy of fusion is taken to be constant at $2.3 \mathrm{R}$. The dilute solution limit $k_{O}$ of the equilibrium partition coefficient of $B$ in $A$ is 0.34 . The solution of eqs. (2a) and (4) for $v=0$, displayed as dashed lines in Fig. $6(a)$, are the solidus and liquidus of the equilibrium phase diagram. The dotted line is the $T_{0}$ curve, which is the locus of the 

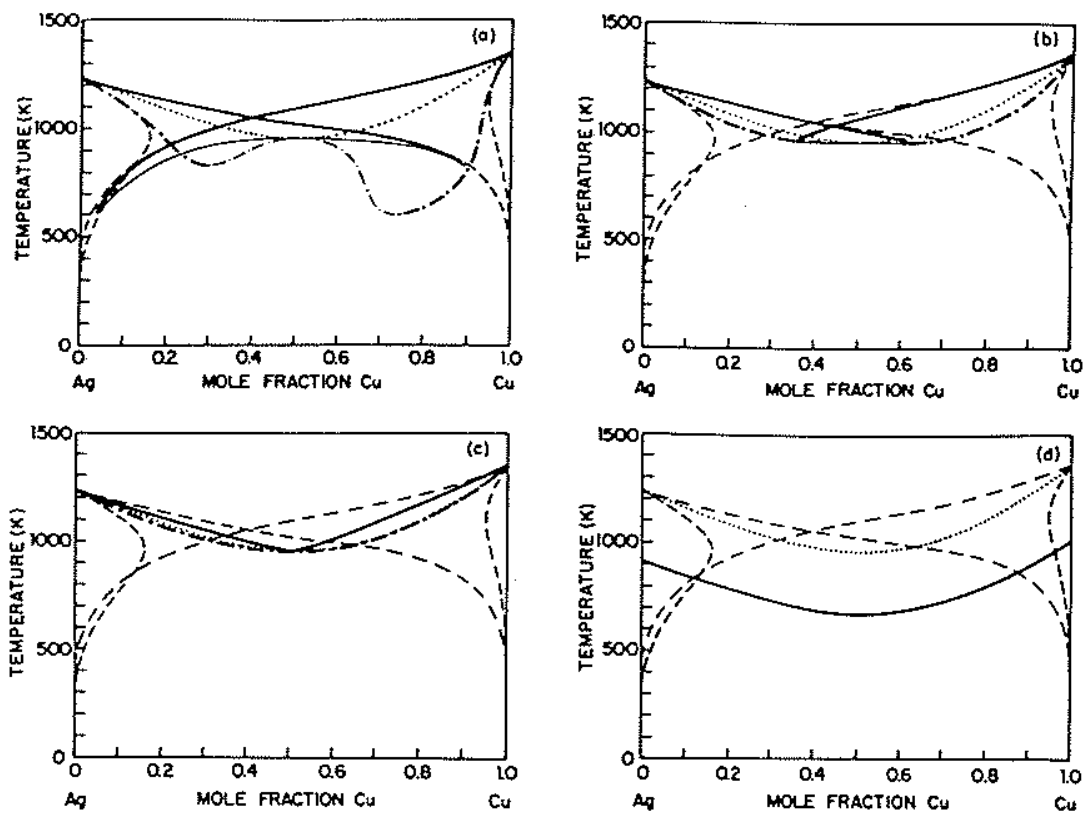

Fig. 8. Kinetic interface condition diagram for Ag-Cu neglecting any effect due to solute drag. Solutions with $v_{O} / v_{D}=100$ for: (a) $v / v_{D}=0.01$; solid line: kinetic liquidus; dot-dash line: kinetic solidus. (b) $v / v_{D}=0.1 ;(c) v / v_{D}=0.7 ;$ (d) $v / v_{D}=30$.

intersections of the solid and liquid free energy curves in Fig. 4. The solid and dot-dashed curves labelled ".1" in Fig. 6(a) are the "kinetic" liquidus and solidus for an imposed interface speed of $0.1 v_{D}$. That is, they are the solutions to egs. (2a) and (4). The development of the theory leading to these diagrams and a more thorough discussion of the diagrams themselves is presented elsewhere [26]. We see that for $v>0$ the liquidus and solidus begin to approach each other, reflecting the increasing partition coefficient (which is the ratio of the kinetic solidus and liquidus compositions). As the velocity is increased, the solidus and liquidus continue to approach each other, as shown in Fig. 6(a). Finally, they merge and drop in temperature as $v$ exceeds $v_{D}$ (not shown).

In Fig. 6(b) are plotted the solutions to equations (2a) and (3) for the same conditions as in Fig. 6(a). We see that in the formulation without the solute drag effect, the kinetic solidus initially rises toward the $T_{o}$ line more rapidly than the kinetic liquidus drops. This can be understood in light of Fig. 5. Eventually, of course, the kinetic solidus and liquidus merge and the interface temperature drops as $v$ exceeds $v_{D}$.

In Figs. 7 and 8 are shown the solutions to eqs. (2a) and (4), and (2a) and (3), respectively, for Murray's themodynamic model of the Ag-Cu system. Again we assume $v_{O} / v_{D}=$ 100. The equilibrium solidi and liquidi are again denoted by dashed curves. Solid-solid equilibrium below the eutectic has been ornitted; metastable solid-liquid equilibria are depicted instead. The solid and dot-dashed curves in Figs. 7 and 8 are the kinetic liquidi and solidi. We see significantly more undercooling predicted in the model with solute drag that in the model with no solute drag. Multiple solutions are found in the middle of the diagrams, e.g. in Fig. 
7(b). This region of the phase diagram lies under the chemical spinodal for the solid and may thus be unobservable experimentally. Since this treatment neglects effects such as coherency strains that tend to stabilize the solid against spinodal decomposition, we have included the "unstable" solutions in the event that some of them might be realized experimentally. Note that Duwez and coworkers apparently produced $\mathrm{Ag}-\mathrm{Cu}$ solid solutions across the entire phase diagram by splat quenching [43]. Representative tie-lines are shown to aid the reading of the diagrams.

An instablility in the steady-state solution occurs where a tie line connects a kinetic solidus and a kinetic liquidus having opposite slopes, such as in the central regions of Figs. 7(b) and 8(a). A fluctuation whereby a small amount of crystal grows with more solute than the steadystate amount will make the liquid at the interface leaner in solute, thus shifting the steady-state solution to yet greater solute concentrations. For similar reasons, a fluctuation in the opposite direction will also tend to grow. This effect could give rise to oscillatory behavior when a constant solidification velocity is imposed on material with a bulk liquid composition in this region [44]. Strictly speaking, the theory yields steady-state response functions only, and any non-steady-state behavior cannot be described in terms of such roots. However, we expect the time it takes for the interface to reach a "steady "state" configuration to be rather short, on the order of $Q^{2} / \mathrm{D}_{\mathrm{i}}$, where $l$ is the width of the interface. Thus the steady state theory may adequately describe unsteady behavior aiso.

Duning rapid regrowth from pulsed laser melting of semiconductors, the interface has been observed to slow down upon encountering a heavily doped region $[45,46]$. The interface velocity was measured in these experiments by the transient electrical conductance technique. Whether this effect is mainly due to solute drag, a reduced driving force for solidification, or a reduced interface mobility $\left(v_{0}\right)$ remains to be seen. This interface velocity measurement technique has recently been successfully applied to metallic thin films [47] as well. In the near future, experiments of this type may enable us to make a quantitative test of the theory for the interface velocity vs. undercooling and composition response function.

\section{SUMMARY}

The transition from diffusion-controlled to diffusionless solidification follows simply and directly from the collision-limited growth model of Turnbull and coworkers, wherein the atomic motions responsible for interface advancement can be much more rapid than those necessary for solute escape at the interface. The continuous growth model describes the measured velocity dependence of trapping of several dopants in Si. The details of the measured orientation and velocity dependence of trapping of $\mathrm{Bi}$ in $\mathrm{Si}$ are understood in terms of $\{111\}$ ledges passing laterally after random intervals, with two opportunities for solute to escape. Quantitative tests of solute trapping models have not yet been made in metals. The interfacial undercooling has been predicted by extending a model developed for pure systems. The resulting interface condition diagrams reproduce the equilibrium phase diagram at zero velocity, and depict interfacial undercooling and suppressed solute partitioning as the interface speed increases. The results are sufficiently different with and without the inclusion of a solute drag term that experiments may be possible to determine whether the solute drag effect is present during solidification of heavily doped semiconductors or of metallic alloys.

Much of the work reviewed here has benefited from the invaluable contributions of my collaborators J.Y. Tsao, M.O. Thompson, P.S. Peercy and C.W. White on the experimental work, and T. Kaplan and L.M. Goldman on the theoretical work. Parts of this research were supported by the Harvard Materials Research Laboratory under contract NSF-DMR-83-16979 and by the U.S. Department of Energy Faculty Research Participation Program administered by Oak Ridge Associated Universities.

\section{REFERENCES}

1. J.C. Baker and J.W. Cahn, Acta Metall. 17, 575 (1969).

2. C.W. White, S.R. Wilson, B.R. Appleton and F.W. Young Jr., J. Appl. Phys. 51, 738 (1980). 

3. P. Baeri, J.M. Poate, S.U. Campisano, G. Foti, E. Rimini, and A.G. Cullis, Appl. Phys.
Letters 37, 912 (1980).

4. J.C. Baker and J.W. Cahn, in Solidification (ASM, Metals Park, Ohio, 1970), pp. 23-58.

5. R.N. Hall, J. Physical Chem. 57, 836 (1953).

6. A.A. Chernov, in Growth of Crystals, edited by A.V. Shubnikov and N.N. Sheftal' (Consultants Bureau, New York, 1962), Vol. 3, p. 35.

7. J.C. Brice, The Growth of Crystals from the Melt (North-Holland, Amsterdam, 1965).

8. J.W. Cahn, S.R. Coriell and W.J. Boettinger, in Laser and Electron Beam Processing of Materials, edited by C.W. White and P.S. Peercy (Academic Press, New York, 1980), p.

9. M. Hillert and B. Sundman, Acta Metall. 25, 11 (1977).

10. K.A. Jackson, in Surface Modification and Alloying by Laser, Ion and Electron Beams, edited by J.M. Poate, G. Foti and D.C. Jacobson (Plenum Press, New York, 1983), p. 51.

11. M.J. Aziz, J. Appl. Phys. 53, 1158 (1982).

12. M.J. Aziz, Appl. Phys. Lett. 43, 552 (1983).

13. G.H. Gilmer, Mat. Res. Soc. Symp. Proc. 13, 249 (1983).

14. D. Turnbull, J. Physical Chem. 66, 609 (1962).

15. D. Tumbull, Contemporary Physics 10, 473 (1969).

16. D. Turnbull, in ref. 4 , pp. 1-22.

17. D. Turnbull, J. de Physique, 35, C4.1 (1974).

18. D. Turnbull and B.G. Bagley, in Treatise on Solid State Chemistry, edited by by N.B. Hannay (Plenum Press, New York, 1975), Vol. 5, pp. 513-54.

19. F. Spaepen and D. Turnbull, in Rapidly Quenched Metals. 2nd International Conference, edited by N.J. Grant and B.C. Giessen (MIT Press, Cambridge, Mass., 1976), pp. 205-29.

20. F. Spaepen and D. Tumbull, in Laser Annealing of Semiconductors, edited by J.M. Poate and J.W. Mayer (Academic Press, New York, 1982), pp. 15-42.

21. D. Turnbull, Metall. Trans. A 12,693 (1981).

22. S.R. Coriell and D. Turnbull, Acta Metall. 30, 2135 (1982).

23. J.Q. Broughton, G.H. Gilmer, and K.A. Jackson, Phys. Rev. Lett. 49, 1496 (1982).

24. C.A. MacDonald, A.M. Malvezzi and F. Spaepen, Mat. Res. Soc. Symp. Proc., edited by H. Kurz, G.L. Olson, and J.M.Poate, 51, 277-282 (1986).

25. L.M. Goldman and M.J. Aziz, submitted to J. Mater. Res.

26. M.J. Aziz and T. Kaplan, submitted to Acta Metall.

27. C.W. White, B.R. Appleton, B. Stritzker, D.M. Zehner and S.R. Wilson, Mat. Res. Soc. Symp. Proc. 1, 59 (1981). 
28. P. Baeri, G. Foti, J.M. Poate, S.U. Campisano and A.G. Cullis, Appl. Phys. Lett. 38, 800 (1981).

29. J.M. Poate, Mat. Res. Soc. Symp. Proc. 4, 121 (1982).

30. C.W. White, D.M. Zehner, S.U. Campisano and A.G. Cullis, in ref, 10, p. 94.

31. M.J. Aziz, Ph.D. thesis, Harvard University (1983), pp. 76-82.

32. M.J. Aziz, J.Y. Tsao, M.O. Thompson, P.S. Peercy and C.W. White, Phys. Rev. Lett. 56, 2489 (1986).

33. M.O. Thompson, G.J. Galvin, J.W. Mayer, P.S. Peercy and R.B. Hammond, Appl. Phys. Lett. 42, 445 (1983).

34. M.J. Aziz, J.Y. Tsao, M.O. Thompson, P.S. Peercy, and C.W. White, Mat. Res. Soc. Symp. Proc. 35, 153 (1985).

35. T. Papa, F. Scudieri, M. Marinelli, U. Zammit, and G. Cembali, J. de Physique, C-5.73 (1983).

36. M.J. Aziz and C.W. White, Phys. Rev. Lett. 57, 2675 (1986).

37. L. Onsager, Phys. Rev. 37, 405 (1931); 38, 2265 (1931).

38. J.W. Christian, in Theory of Transformations in Metals and Alloys, Part I, 2nd ed. (Pergamon, Oxford, 1975), p. 479.

39. W.J. Boettinger, S.R. Coriell and R.F. Sekerka, Mat. Sci. and Eng. 65, 27 (1984).

40. J.W. Cahn, Acta Metall. 10, 789 (1962).

41. J.L. Murray, Metall, Trans. A 15,261 (1984).

42. W.J. Boettinger, D. Shechtman, R.J. Schaefer, F. Biancaniello, Metall. Trans. A 15, 55 (1984).

43. P. Duwez, R.H. Willens, and W. Klement, J. Appl. Phys. 31, 1136 (1960).

44. M.J. Aziz, in Hume-Rothery Symposium on Undercooled Alloy Phases, edited by E.W. Collings and C.C. Koch, TMS-AIME Symp. Proc. (1986).

45. G.J. Galvin, J.W. Mayer and P.S. Peercy, Mat. Res. Soc. Symp. Proc. 23, 111 (1984).

46. P.S. Peercy and M.O. Thompson, Mat. Res. Soci. Symp. Proc. 35, 53 (1985).

47. J.Y. Tsao, S.T. Picraux, P.S. Peercy, and M.O. Thompson, Appl. Phys. Lett. 48, 278 (1986). 